had active disease. CDUS findings were: synovial proliferation: grade $1: 27.4 \%$, grade 2: $54.7 \%$, grade 3: $11.6 \%$; vascularity: grade 1: $41.1 \%$, grade 2: $32.6 \%$ and grade $3: 9.5 \%$. Summary findings of QUS were: $C F(I S): 8.6 \pm 10.63$ (median 4.4), $\mathrm{CF}$ (total): $9.9 \pm 11$ (median 5.2), median $\mathrm{N}(\mathrm{IS}): 3(0-26)$ and mean $\mathrm{N}(\mathrm{ES})$ : $1.2(0-19)$. Cohen's kappa for synovial proliferation was $0.402(p<0.001)$ and vascularity was $0.605(p<0.001)$. Intraclass correlation $(\operatorname{ICC}(1,2))$ for $\mathrm{CF}(\mathrm{IS})$ was 0.996 (95\% confidence interval (Cl): 0.994-0.997) and ICC $(1,2)$ for $\mathrm{CF}$ (total) was 0.995 (95\% Cl: 0.993-0.997). CF(IS) was correlated with SJC ( $r=0.22, p=0.029)$, TJC $(r=0.39, p<0.001)$, PGA $(r=0.5, p<0.001)$, EGA $(r=0.583, p<0.001)$ and DAS28 $(r=0.47, p<0.001)$; correlations for $C F$ (total) were: $S J C(r=0.25, p=0.013)$, TJC $(r=0.41, p<0.001)$, PGA $(r=0.525, p<0.001)$, EGA $(r=0.515, p<0.001)$ and DAS28 $(r=0.5, p<0.001)$. Significant correlations were also observed for $N(I S)$ with SJC $(r=0.282, p=0.006)$, TJC $(r=0.411, p<0.001), P G A(r=0.48, p<0.001)$, EGA $(r=0.514, p<0.001)$ and DAS28 $(r=0.467, p<0.001)$. Spearman rank correlations of CDUS vascularity were: SJC $(r=0.26, p=0.01)$, TJC $(r=0.292, p=0.004)$, PGA $(r=0.26, p=0.012)$, EGA $(r=0.168, p=0.103)$ and DAS28 $(r=0.275, p=0.007)$. Spearman rank correlations of $\mathrm{CF}(\mathrm{IS}), \mathrm{CF}$ (total) and $\mathrm{N}(\mathrm{IS})$ with CDUS vascularity were $0.828,0.864$ and 0.689 respectively $(p<0.001)$. Cut-off values for $C F(I S)$, $\mathrm{CF}$ (total) and $\mathrm{N}(\mathrm{IS})$ for distinguishing active RA from RA in remission were 4.78 (AUC: $0.82,95 \% \mathrm{Cl}: 0.73-0.9$ ), 5.75 (AUC: $0.89,95 \% \mathrm{Cl}: 0.69-0.88$ ) and 2.5 (AUC: $0.77,95 \% \mathrm{Cl}: 0.68-0.86$ ) respectively. There were 40 patients with CDUS vascularity $\geq 2$ among which $62.5 \%(25 / 40)$ had active disease. In this group only $\mathrm{CF}$ (total) $>5.75$ could distinguish between patients with active disease from disease in remission $(100 \%$ (25/25) vs., $80 \%$ (12/25), $p=0.046)$.

Conclusions: $\mathrm{CF}(\mathrm{IS})$ and $\mathrm{CF}$ (total) had excellent inter-rater reliability and construct validity. Simple quantitative cutoff values could distinguish between active RA from remission.

Disclosure of Interest: None declared

DOI: 10.1136/annrheumdis-2017-eular.6482

\section{AB1055 LEUKOCYTE ESTERASE REAGENT STRIPS FOR RAPID DIAGNOSIS OF INFLAMMATORY SYNOVIAL FLUID}

S. Rodríguez-Muguruza ${ }^{1}$, S. Malumbres ${ }^{2}$, A. Olive $^{3}$, O. Valero ${ }^{4}$, S. Holgado ${ }^{3}$, L. Mateo ${ }^{3}$, M. Martínez-Morillo ${ }^{3} \cdot{ }^{1}$ Rheumatology, Virgen de la Cinta Hospital, Tarragona, Spain; ${ }^{2}$ Clinical Biochemistry Service, Germans Trias I Pujol Hospital, Barcelona, Belgium; ${ }^{3}$ Rheumatology, Germans Trias I Pujol Hospital;

${ }^{4}$ Department of Statistics, Automona de Barcelona University, Barcelona, Spain

Background: The analysis of synovial fluid is an important tool for diagnosing joint disease. When synovial fluid is removed, the white cell count (WCC) decreases with time, and an inflammatory liquid could become a false non-inflammatory specimen. Reagent strip testing of urine is a valid tool for the diagnosis of urinary tract infection, via the detection of leukocyte esterase activity. It has been used for the analysis of others body fluids. Synovial fluid test at the site of arthrocentesis using reagent strips could have potential benefits as a screening tool.

Objectives: To evaluate the performance of leukocyte esterase reagent strips for diagnosis of inflammatory synovial fluid.

Methods: Prospective single center study. We analyzed synovial fluids samples collected from patients in a tertiary university Hospital (November 2015- December 2016). Synovial fluid samples were tested within 1 hour after collection. We analyzed: The presence of leukocyte esterase using the leukocyte esterase reagent strips test (originally designed for urine test, URI-Clip Test, Menarini Diagnostics). It was recorded semi quantitatively: negative, $1+(>25 \mathrm{WBC} / \mathrm{uL}), 2+$ ( $>75 \mathrm{WBC} / \mathrm{uL}$ ) or $3+(>500 \mathrm{WBC} / \mathrm{uL}$ ) by comparison with a standard color chart found on the container's label. The WCC, formula, glucose level. The WCC was measured by manual leukocyte counting, using saline as diluents. Cultures were also collected. We consider + if leucocyte esterase pad was more or equal than $1+$ positive. The cut-off for the WCC $\left(>2000 \mathrm{cells} / \mathrm{mm}^{3}\right)$ was used to differentiate between inflammatory and non-inflammatory specimens. We compared the WCC (reference standard diagnostic test) with the presence of leukocyte esterase using the leukocyte esterase reagent. Sensitivity (Se), specificity (Sp), PPV, NPV were determined. P-value smaller than 0.05 were considered significant.

Results: During the study period, 125 joint fluid specimens were analyzed: 56 $(44.8 \%)$ mechanical and $69(55.2 \%)$ inflammatory. Of the mechanical fluids 33 $(58.9 \%)$ were negative by leukocyte esterase reagent and of the inflammatory fluids $67(97.1 \%)$ were positive. The Se and Sp of leukocyte esterase reagent was $97.1 \%$ and $58.9 \%$ respectively. The PPV was $74.4 \%$ and NPV was $94.3 \%$. The 2 false-negative results (negative by leukocyte esterase reagent but more than $2000 \mathrm{WBC} / \mathrm{mm}^{3}$ ), showed a predominance of mononuclears (> $\left.=91 \%\right)$, the median WCC was $2775 / \mathrm{mm}$ and median neutrophil percentage was $8.5 \%$. For inflammatory fluids: semi-quantitative results (negative, $1+, 2+$ and $3+$ ) were significantly different regarding the main leukocyte, neutrophil and lymphocyte count (table).

\begin{tabular}{lccccc}
\hline & Negative & $1+$ & $2+$ & $3+$ & p value \\
\hline Total cell $\left(\mathrm{cel} / \mathrm{mm}^{3}\right)$ & 3025 & 18272.9 & 10553.5 & 56494.2 & $<0.0001$ \\
Leukocyte $\left(\mathrm{cel} / \mathrm{mm}^{3}\right)$ & 2775 & 12907.1 & 8528.8 & 30870.2 & $<0.0001$ \\
Lymphocyte $(\%)$ & 91.5 & 50.4 & 21.61 & 6.4 & $<0.0001$ \\
Neutrophil $(\%)$ & 8.5 & 49.6 & 83.7 & 91.7 & $<0.0001$ \\
\hline
\end{tabular}

Conclusions: Our results demonstrate that leukocyte esterase reagent strips are a rapid, cheap, and sensitive tool to identify inflammatory synovial fluid. Leukocyte esterase reagent strips had an excellent Se but a poor $\mathrm{Sp}$, it could be used as a screening tool in primary care practice. A positive result may indicate an inflammatory process, then the patient should be referred to a rheumatologist.

Disclosure of Interest: None declared

DOI: 10.1136/annrheumdis-2017-eular.2657

\section{AB1056 RADIOGRAPHIC ANALYSIS OF METATARSUS PRIMUS ELEVATUS IN PATIENTS WITH RHEUMATOID FOREFOOT DEFORMITIES}

S. Mizuki, D. Hiraoka, K. Kushimoto, H. Yamasaki, K. Yoshida, K. Oryoji, K. Kamada, E. Yokota. The Center for Rheumatology, Matsuyama Red Cross Hospital, Matsuyama, Ehime, Japan

Background: Metatarsus primus elevatus involves dorsal elevation of the first metatarsal relative to the lesser metatarsals. The role of metatarsus primus elevatus in rheumatoid forefoot deformity is yet to be elucidated. We hypothesize that metatarsus primus elevatus can be attributed to a different pathomechanism than typical rheumatoid forefoot deformities such as hallux valgus, splay foot, and flat foot.

Objectives: To clarify the radiographic characteristics of metatarsus primus elevatus in patients with rheumatoid forefoot deformities.

Methods: We retrospectively reviewed standing anteroposterior and lateral radiographs of 51 feet (37 patients; mean age $65.7 \pm 7.1$ years) before toeplasty due to metatarsalgia at our hospital. The elevation of the first metatarsal relative to the second metatarsal (MPE), the hallux valgus angle (HVA), the intermetatarsal angle (IMA), talar pitch, and calcaneal pitch were measured. For statistical analyses, the Mann-Whitney $U$ test was used.

Results: Median MPE was $1.7 \mathrm{~mm}$ (interquartile range: $0.7-4.5 \mathrm{~mm}$ ). We compared the group with MPE $>5 \mathrm{~mm}(\mathrm{~N}=12$ feet) to the group with MPE $\leq 5$ $\mathrm{mm}(\mathrm{N}=39$ feet). The group with higher MPE was significantly younger $(\mathrm{p}=0.01 \overline{1})$. There was no significant difference in HVA between the two groups $(p=0.068)$, although IMA was significantly smaller in the group with higher MPE $(p=0.033)$. In the group with higher MPE, calcaneal pitch was greater $(p<0.001)$ and talar pitch was smaller $(p=0.016)$.

Conclusions: In patients with metatarsus primus elevatus, other rheumatoid forefoot deformities such as spray foot and flat foot were not observed. There was a wide range of hallux valgus severity. Metatarsus primus elevatus may be attributed to greater hindfoot calcaneal pitch.

Disclosure of Interest: None declared

DOI: 10.1136/annrheumdis-2017-eular.1945

\section{AB1057 MULTIDISCIPLINARY APPROACH FOR DIAGNOSING CONNECTIVE TISSUE DISEASE-RELATED LUNG DISEASE: IS IT USEFUL FOR RHEUMATOLOGISTS?}

S. Peña Montelongo ${ }^{1}$, A. Monroy Calero ${ }^{1}$, O. Acosta Fernández ${ }^{2}$, S. García Hernández $^{3}$, L. Pérez Martín ${ }^{2}$, A. Bonilla Arjona ${ }^{4}$, H. Sánchez Pérez ${ }^{1}$,

S. Bustabad Reyes ${ }^{1}$, B. Rodríguez-Lozano ${ }^{1} .{ }^{1}$ Rheumatology; ${ }^{2}$ Pneumology;

${ }^{3}$ Pathology; ${ }^{4}$ Chest Section, Radiology, Hospital Universitario de Canarias,

Tenerife, Spain

Background: Many patients with idiopathic interstitial pneumonia (IP) have certain clinical, serological and/or pulmonary morphologic features suggesting an underlying autoimmune disease (AD), but without meeting established criteria for connective tissue disease (CTD), a situation labelled as "IP with autoimmune features" (IPAF) ${ }^{1}$. To identify an underlying aetiology is important because it may impact on treatment and prognosis, and could be optimised by multidisciplinary approach.

Objectives: 1.To identify prevalence of IPAF in routine practice. 2. To determine the value of lung biopsy to diagnose underlying AD.3. To describe the course of IPAF.

Methods: Observational, longitudinal retrospective study in a tertiary hospital specific outpatient clinic for IP patients with cough and dyspnea as dominant symptoms requiring co-evaluation by rheumatologists in 2010-2016. Variables included: clinical, serologic and morphological findings by High-Resolution Computed Tomography of lungs assessed by 2011 current guidelines ${ }^{2}$ and open lung biopsy. Statistical analysis:SPSS 17.0.

Results: Of 410 patients evaluated for IP, 93 had rheumatologist assessment, 70 $\mathrm{F}(75.3 \%)$, mean age at diagnosis 62.6 years (SD12.13), of whose 48 had no previous diagnosis. Mean follow-up 3.54 (SD2.77)years in undiagnosed patients. The most frequent radiological patterns were: inconsistent with usual interstitial pneumonia (UIP) $(67.7 \%)$, UIP $(22.6 \%)$, Possible UIP $(4.3 \%)$, others $(4.3 \%)$. Lung biopsy was performed in 15 patients (16\%), 11 without previous diagnosis. Histopathology patterns: 8 non-specific interstitial pneumonia (NSIP) in whom final rheumathologic clinical diagnosis was IPAF in 6 and UCTD in 2; 1 UIP (Sjögren); 1 vasculitis ( $p$-ANCA) and 1 lymphoid interstitial pneumonia (LIP) corresponding to IPAF. We found lymphoid aggregates in one patient diagnosed as a IPAF. Overall new diagnostics were: IPAF 43.8\%, UCTD 4.2\%, CTD 52.1\%.14/93 (15\%) patients died, of whom 6 had IPAF (43\%).

Conclusions: 1 . Of patients with IP referred for rheumatologist assessment, $31 \%$ has no established CTD, with IPAF as clinical diagnosis in $24.7 \%$ of overall patients. 2. Surgical lung biopsy allowed to diagnose AD in $23 \%$ of unlabelled 